\title{
Influência do Tabagismo no Sistema Vascular Materno-fetal: Estudo com Dopplervelocimetria
}

\author{
Effect of Cigarette Smoking on Maternal-fetal Blood Flow: Doppler Velocimetry Study
}

\author{
Gilberto Rodrigues Pinto, Clovis Botelho
}

\begin{abstract}
RESUM0
Objetivo: avaliar a influência do tabagismo materno na dinâmica vascular materno-fetal, por meio da dopplervelocimetria obstétrica das artérias uterinas, umbilical e cerebral média. Métodos: estudo prospectivo em 42 gestantes saudáveis, sendo 20 fumantes e 22 não-fumantes. Foram realizadas ultra-sonografias para determinar o tempo de gestação e exame de dopplervelocimetria pulsátil das artérias uterinas, umbilical e cerebral média nas $24^{a}$, $28^{a}$, $32^{\underline{a}}$ e $36^{a}$ semana para avaliar o fluxo vascular materno-fetal. As fumantes foram orientadas a não fumar por pelo menos 2 horas antes do exame.

Resultados: o índice de resistência (IR) médio das artérias uterinas $D$ e E foi maior no grupo de fumantes na $36^{a}$ semana com média de 0,50 e desvio padrão de 0,034. O índice de pulsatilidade (IP) da artéria umbilical foi maior no grupo de fumantes na $28^{a}$ semana $[\bar{x}(D P)=1,135(0,182)]$, $p=0,008$; o IP da artéria cerebral média (ACM) não se mostrou diferente entre os grupos, porém, a relação IP ACM/ umbilical foi menor no grupo de fumantes, na $32^{a}$ semana $[\bar{x}(D P)=$ $1,9(0,291)], p=0,027$ e na $36^{a}$ semana $[\bar{x}(D P)=1,850(0,465)], p=0,014$.

Conclusões: os índices de dopplervelocimetria mostraram que no grupo de fumantes há aumento na resistência da circulação útero-placentária e feto-placentária, associado a concomitante diminuição na resistência da ACM, mimetizando uma tendência do tabagismo levar à hipóxia crônica do feto.
\end{abstract}

PALAVRAS-CHAVE: Tabagismo. Dopplervelocimetria. Gravidez normal.

\section{Introdução}

O uso do fumo durante o ciclo gravídicopuerperal resulta em diversas conseqüências, porém, o seu mecanismo fisiopatológico ainda não está totalmente elucidado ${ }^{1}$. É descrita maior incidência de fetos pequenos para a idade gestacional entre pacientes fumantes. Recentemente, Castles et $\mathrm{al}^{2}$, em amplo estudo de meta-análise, reviu e confirmou a forte associação do tabagismo com um elevado risco de placenta prévia, descolamento prematuro de placenta, gravi-

Trabalho realizado no Hospital Universitário Júlio Muller/ UFMT, com suporte financeiro do CNPq.

Correspondência:

Clovis Botelho

Rua Dr. Jonas Correa da Costa, 210

78.030-510 - Cuiabá/MT

Fone: (65) 637-1471 / Fax: (65) 637-7539

e-mail: fbotelho@nutecnet.com.br dez ectópica e ruptura prematura de membrana, e um risco diminuído para pré-eclâmpsia.

A dopplervelocimetria na gestação, por sua ampla aplicação clínica, devido à facilidade do seu manuseio e da inocuidade para a paciente, torna-se extremamente importante para se avaliarem alterações vasculares decorrentes das mais variadas causas, dentre elas as relacionadas com o tabagismo.

A nicotina e o monóxido de carbono são as substâncias que mais provavelmente causam dano no sistema vascular materno-fetal. Os efeitos da nicotina no sistema cardiovascular, de modo geral, imitam aqueles vistos após ativação do sistema simpático adrenal, incluindo efeitos inotrópico e cronotrópico positivos no miocárdio, bem como aumento do rendimento cardíaco. Além disso, as pressões sistólica e diastólica aumentam secundariamente à estimulação do eixo 
simpático adrenal ${ }^{3}$.

O monóxido de carbono difunde-se lentamente pela placenta e o nível de carboxihemoglobina fetal é 10 a $15 \%$ mais elevado que o materno. Taxas aumentadas de carboxihemoglobina no concepto, por seus efeitos na curva de dissociação da oxi-hemoglobina, estão associadas com diminuição na pressão parcial de oxigênio no sangue ${ }^{4}$.

Este trabalho tem como objetivo avaliar a influência do tabagismo sobre o sistema vascular materno-fetal, por meio da dopplervelocimetria das artérias uterinas, umbilical e cerebral média de gestantes normais, com a finalidade de estabelecer quantitativamente tais alterações, para servir como prognóstico para o feto da gestante fumante.

\section{Pacientes e Métodos}

Foram estudadas de forma prospectiva 42 gestantes normais atendidas no Ambulatório de Pré-natal do Departamento de Ginecologia e Obstetrícia, da Faculdade de Ciências Médicas, da Universidade Federal de Mato Grosso, cujo funcionamento ocorre no Hospital Universitário Júlio Müller (HUJM), em Cuiabá/MT, no período de dezembro de 1997 a janeiro de 1999.

Das 42 pacientes que iniciaram a pesquisa duas não tiveram seus dados computados devido a complicações obstétricas. Uma gestante era fumante e apresentou um descolamento prematuro de placenta, com 28 semanas, evoluindo para óbito fetal, e seus níveis pressóricos eram normais. Outra paciente, não-fumante, apresentou quadro de pré-eclâmpsia grave no 3 - trimestre de gestação.

Não houve diferença estatisticamente significante entre as médias de idade, número de gestações anteriores, paridade e número de abortos entre os dois grupos de gestantes (respectivamente, $p=0,433 ; p=0,909 ; p=0,713$ e $p=$ 0,793), mostrando que os dois grupos são comparáveis em relação a estas variáveis (Tabela 1).

As gestantes foram agrupadas conforme $o$ hábito tabagístico, ficando 20 gestantes no grupo chamado de fumantes e 22 no grupo de nãofumantes. Os critérios de inclusão foram: terem iniciado o pré-natal antes da $20^{\mathrm{a}}$ semana de gestação e serem voluntárias para participarem da pesquisa.

A quantificação do hábito tabagístico foi a seguinte: 3 pacientes referiram fumar regularmente de 1 a 5 cigarros por dia, 7 fumavam de 6 a 10 cigarros e 9 referiram fumar de 11 a 20 ci- garros por dia. Devido ao pequeno tamanho amostral a categorização do grupo de fumantes, pelo consumo diário de cigarros, não foi considerada para análise.

Tabela 1 - Distribuição das gestantes conforme idade, número de gestações (gesta), partos (para) e abortos, segundo o hábito tabagístico. (teste \pm de student)

\begin{tabular}{lccc}
\hline Variável & $\begin{array}{c}\text { Fumantes } \\
\overline{\mathbf{x}}(\mathbf{D P}) \\
(\mathbf{n}=\mathbf{1 9})\end{array}$ & $\begin{array}{c}\text { Não-fumantes } \\
\overline{\mathbf{x}}(\mathbf{D P})\end{array}$ & $\mathbf{p}$ \\
$\mathbf{( n = 2 1 )}$ & \\
\hline Idade & $24,4(4,1)$ & $23,4(3,8)$ & 0,433 \\
Min-max & $18-32$ & $16-30$ & \\
Gesta & $2,5(1,3)$ & $2,5(1,4)$ & 0,909 \\
Min-max & $1-5$ & $1-6$ & \\
Para & $1,1(1,0)$ & $1,0(0,9)$ & 0,713 \\
Min-max & $0-3$ & $0-3$ & \\
Aborto & $0,4(0,7)$ & $0,4(0,7)$ & 0,793 \\
Min-max & $0-2$ & $0-2$ & \\
\hline
\end{tabular}

$\bar{x}(D P)=$ média (desvio-padrão)

Foram considerados critérios de exclusão: a) ser portadora de alguma doença previamente conhecida; b) estar em uso regular de medicação, com exceção de vitaminas; c) ter apresentado quadro clínico e/ou sorologia compatível com rubéola, toxoplasmose, sífilis e citomegalovírus nesta gravidez; d) desenvolver durante o pré-natal sindrome hipertensiva ou diabetes gestacional; e) ser portadora de feto malformado ou com cromossomopatia; f) apresentar gestação múltipla.

Os exames de ultra-sonografia obstétrica e dopplervelocimetria foram realizados em aparelho marca Siemens, modelo Sonoline Versa Pro com mapeamento em cores, com Doppler pulsátil e transdutor convexo de $4,0 \mathrm{MHz}$, por um único examinador.

A idade gestacional foi avaliada com ultrasonografia até 20 semanas de gestação. Caso houvesse diferença de até uma semana entre a idade gestacional calculada pela data da última menstruação e aquela dada pela ultra-sonografia, foi considerada a primeira. Havendo diferença maior considerou-se a idade ultra-sonográfica.

As pacientes foram encaminhadas para realização de exame de dopplervelocimetria obstétrica com 24, 28, 32, 36 e 40 semanas de gestação. Para este estudo não foram considerados os resultados da $40^{\text {a }}$ semana, pelo baixo índice de comparecimento, pois a grande maioria das gestantes já haviam dado à luz, o que impossibilitou a análise destes dados. 
As pacientes, ao chegarem para o exame, eram inicialmente atendidas por uma secretária que as colocava na sala de ultra-sonografia. $O$ exame era realizado sem conhecimento pelo pesquisador do hábito tabagístico das mesmas.

As gestantes fumantes receberam recomendação para não fumar por pelo menos 2 horas antes do horário marcado para a realização do exame Doppler, para evitar os efeitos agudos do tabagismo sobre os parâmetros da dopplervelocimetria.

A dopplervelocimetria foi realizada com a gestante em decúbito semilateral, para todas as artérias, que foram identificadas usando equipamento de Doppler duplex, com mapeamento em cores, com transdutor convexo de 4,0 MHz. As medidas foram obtidas pela média de 3 medições diferentes para cada vaso. Para todos os vasos o ângulo entre o cursor e o vaso não excedeu 30 graus.

As seguintes variáveis foram consideradas para este estudo: a) média dos índices de resistência (IR) das artérias uterinas direita e esquerda (UTM); b) índice de pulsatilidade (IP) da artéria umbilical (Umb) c) índice de pulsatilidade da artéria cerebral média $(\mathrm{ACM})$; d) relação índice de pulsatilidade da artéria cerebral média/índice de pulsatilidade da artéria umbilical (IP ACM/ $\mathrm{Umb}$ ).

Foi realizada a análise de variância a um fator utilizando o teste $t$ de Student ou o teste $\mathrm{U}$ de Mann-Whitney. Foram elaborados tabelas que mostram os valores das médias e seus respectivos intervalos de confiança (95\%). Optou-se por trabalhar com a distribuição $t$ de Student, pois as variâncias populacionais eram desconhecidas e os tamanhos das amostras em cada grupo eram diferentes e pequenos. Foi considerado erro alfa de $5 \%$.

Este estudo não apresentou riscos às gestantes ou ao concepto, sendo realizado mediante aprovação da Comissão de Ética do HUJM.

\section{Resultados}

Na Tabela 2 vêem-se os resultados da análise das médias dos IR das artérias uterinas direita e esquerda em cada semana gestacional estudada, segundo o hábito tabagístico. Verifica-se que houve diferença estatisticamente significante entre as médias de UTM na $36^{\mathrm{a}}$ semana $(p=0,002)$, sendo que o grupo de gestantes fumantes teve média de UTM superior $[0,501$ $(0,034)]$ à do grupo de não fumantes $[0,452$ $(0,037)]$. Nas outras semanas de estudo não hou- ve diferença estatisticamente significante entre as médias dos dois grupos.

Tabela 2 - Médias dos índices de resistência entre as artérias uterinas direita e esquerda, nas diversas semanas de estudo, segundo o hábito tabagístico. (teste U de Mann-Whitney)

\begin{tabular}{|c|c|c|c|}
\hline $\begin{array}{l}\text { Semana de } \\
\text { Gestação }\end{array}$ & $\begin{array}{c}\text { Fumantes } \\
\overline{\bar{x}}(\mathrm{DP})\end{array}$ & $\begin{array}{c}\text { Não-fumantes } \\
\bar{x}(\mathrm{DP})\end{array}$ & $\mathbf{P}$ \\
\hline $24^{a}$ & $\begin{array}{c}0,534(0,054) \\
(n=16)\end{array}$ & $\begin{array}{c}0,523(0,073) \\
(n=17)\end{array}$ & 0,718 \\
\hline $28^{a}$ & $\begin{array}{c}0,538(0,059) \\
(n=17)\end{array}$ & $\begin{array}{c}0,507(0,055) \\
(n=19)\end{array}$ & 0,193 \\
\hline $32^{a}$ & $\begin{array}{c}0,500(0,049) \\
(n=14)\end{array}$ & $\begin{array}{c}0,470(0,044) \\
(n=14)\end{array}$ & 0,093 \\
\hline $36^{a}$ & $\begin{array}{c}0,501(0,034) \\
(n=14)\end{array}$ & $\begin{array}{c}0,452(0,037) \\
(n=15)\end{array}$ & 0,002 * \\
\hline
\end{tabular}

Para a artéria umbilical foi considerado o IP, estando os seus resultados na Tabela 3 . Verifica-se que o grupo de fumantes teve média de IP superior $[1,135(0,182)]$ à do grupo de nãofumantes $[0,999(0,098)]$, na $28^{a}$ semana $(p=$ $0,008)$. Nas outras semanas de estudo não houve diferença estatisticamente significante entre as médias dos dois grupos.

Tabela 3 - Médias do índice de pulsatilidade da artéria umbilical, nas diversas semanas de estudo segundo o hábito tabagístico.

\begin{tabular}{|c|c|c|c|}
\hline $\begin{array}{l}\text { Semana de } \\
\text { Gestação }\end{array}$ & $\begin{array}{l}\text { Fumantes } \\
\begin{array}{l}\bar{x}(D P) \\
(n=17)\end{array}\end{array}$ & $\begin{array}{c}\text { Não-fumantes } \\
\begin{array}{c}\bar{x}(D P) \\
(n=21)\end{array}\end{array}$ & $p$ \\
\hline $24^{a}$ & $\begin{array}{c}1,156(0,165) \\
(n=18)\end{array}$ & $\begin{array}{c}1,095(0,118) \\
(n=21)\end{array}$ & $0,196^{t}$ \\
\hline $28^{a}$ & $\begin{array}{c}1,135(0,182) \\
(n=18)\end{array}$ & $\begin{array}{c}0,999(0,098) \\
(n=19)\end{array}$ & $0,008^{U^{*}}$ \\
\hline $32^{\mathrm{a}}$ & $\begin{array}{c}0,963(0,175) \\
(n=17)\end{array}$ & $\begin{array}{c}0,922(0,099) \\
(n=19)\end{array}$ & $0,704^{\mathrm{U}}$ \\
\hline $36^{a}$ & $0,866(0,159)$ & $0,802(0,113)$ & $0,169^{t}$ \\
\hline
\end{tabular}

Na Tabela 4 estão apresentados os resultados do estudo da ACM. Verifica-se que não houve diferença estatisticamente significante entre as médias do IP da ACM nos dois grupos de estudo, em todas as semanas de gestação estudadas. 
Tabela 4 - Médias do índice de pulsatilidade da artéria cerebral média, nas diversas semanas de estudo, segundo o hábito tabagístico (teste te Student).

\begin{tabular}{lccc}
\hline $\begin{array}{l}\text { Semana de } \\
\text { Gestação }\end{array}$ & $\begin{array}{c}\text { Fumantes } \\
\overline{\mathbf{x}}(\mathrm{DP}) \\
(\mathbf{n}=17)\end{array}$ & $\begin{array}{c}\text { Não-Fumantes } \\
\overline{\mathbf{x}}(\mathrm{DP})\end{array}$ & $\mathbf{p}$ \\
& $(\mathbf{n}=\mathbf{2 1})$ & \\
\hline $24^{\underline{a}}$ & $2,014(0,720)$ & $2,181(0,708)$ & 0,477 \\
& $(n=18)$ & $(n=21)$ & \\
$28^{\underline{a}}$ & $2,111(0,525)$ & $2,061(0,466)$ & 0,754 \\
& $(n=18)$ & $(n=19)$ & \\
$32^{\underline{a}}$ & $1,872(0,248)$ & $2,034(0,287)$ & 0,075 \\
& $(n=17)$ & $(n=19)$ & \\
$36^{\underline{a}}$ & $1,561(0,320)$ & $1,752(0,287)$ & 0,068 \\
\hline$\overline{\bar{x}}$ (DP) = média (desvio-padrão) & &
\end{tabular}

A Tabela 5 apresenta a análise dos resultados referentes à relação do índice de pulsatilidade da ACM e da artéria umbilical. Verifica-se que houve diferença estatisticamente significante entre as médias de IP ACM/Umb na $32^{\text {a }}$ e $36^{\text {a }}$ semana de estudo (respectivamente, $\mathrm{p}=0,027 \mathrm{e}$ $\mathrm{p}=0,014)$, sendo para o grupo de fumantes a média e desvio padrão na $32^{\text {a }}$ semana de 1,977 $(0,291)$ e de $1,850(0,465)$ na $36^{\text {a }}$ semana, e para o grupo de não-fumantes os valores respectivos na $32^{\text {a }}$ e $36^{\text {a }}$ semana foram $2,224(0,350)$ e 2,206 $(0,354)$.

Tabela 5 - Médias da relação entre o IP da artéria cerebral média e o IP da artéria umbilical (IP ACM/Umb) nas diversas semanas de estudo, segundo o hábito tabagístico (testet de Student).

\begin{tabular}{|c|c|c|c|}
\hline $\begin{array}{l}\text { Semana de } \\
\text { Gestação }\end{array}$ & $\begin{array}{l}\text { Fumantes } \\
\qquad \begin{array}{c}\bar{x}(D P) \\
(n=17)\end{array}\end{array}$ & $\begin{array}{l}\text { Não Fumantes } \\
\begin{array}{c}\bar{x}(D P) \\
(n=21)\end{array}\end{array}$ & $p$ \\
\hline $24^{a}$ & $\begin{array}{c}1,773(0,690) \\
(n=18)\end{array}$ & $\begin{array}{c}2,019(0,707) \\
(n=21)\end{array}$ & 0,289 \\
\hline $28^{a}$ & $\begin{array}{c}1,909(0,572) \\
(n=18)\end{array}$ & $\begin{array}{c}2,096(0,592) \\
\quad(n=19)\end{array}$ & 0,323 \\
\hline $32^{a}$ & $\begin{array}{c}1,977(0,291) \\
(n=17)\end{array}$ & $\begin{array}{c}2,224(0,350) \\
(n=19)\end{array}$ & $0,027^{*}$ \\
\hline $36^{\underline{a}}$ & $1,850(0,465)$ & $2,206(0,354)$ & $0,014^{*}$ \\
\hline
\end{tabular}

\section{Discussão}

Os resultados aqui apresentados confirmam a influência do tabagismo na gestação, que causou padrão de perfusão materno-fetal com características de hipóxia crônica. O estudo dessa dinâmica vascular na gestante fumante pode contribuir para o entendimento de como o tabagismo materno está associado com maior morbidade fetal e neonatal.

Alguns estudos já foram feitos para detectar os efeitos do tabaco, sobre os parâmetros de dopplervelocimetria obstétrica. Eriksen e $\mathrm{Marsal}^{5}$ estudaram gestantes fumantes antes e imediatamente após fumarem um cigarro e constataram aumento da freqüência cardíaca e da pressão arterial materna, além de aumento da freqüência cardíaca fetal. Paralelamente, observaram significante aumento transitório do fluxo sangüíneo na veia umbilical e aorta fetal.

Morrow et al. ${ }^{6}$, com método semelhante, confirmaram os achados acima citados e, ainda, observaram aumento da relação S/D na artéria umbilical após o fumo. Seus resultados não encontraram alteração na relação S/D da artéria uterina, concluindo que o tabagismo causa considerável mudanças na circulação feto-placentária, que são similares àquelas no retardo de crescimento fetal, sendo indicativos de resistência vascular placentária aumentada. Lymperopoulou et al. $^{7}$ observaram aumento significativo de resistência na artéria uterina direita e na artéria umbilical, paralelamente ao aumento de cotinina plasmática, e concluem que a associação entre tabagismo e morbidade perinatal, muito provavelmente, envolve aumento de resistência vascular do útero e placenta.

Todos os estudos baseados no efeito agudo do cigarro encontraram aumento da pressão arterial e da freqüência cardíaca materna, associado a aumento da freqüência cardíaca fetal. Tais alterações hemodinâmicas influenciam a velocidade de fluxo das artérias uterinas e umbilical, dificultando uma melhor interpretação da ação do tabagismo sobre o sistema vascular materno-fetal.

Para afastar os efeitos agudos do cigarro na circulação materno-fetal, como aumento da pressão arterial e da freqüência cardíaca materna e fetal, os exames de dopplervelocimetria foram realizados no mínimo duas horas após o uso do último cigarro; assim, poder-se-ia imputar os resultados encontrados aos efeitos crônicos do tabagismo, que é a proposta deste estudo ${ }^{8}$.

Kimya et al. ${ }^{9}$ estudaram 22 gestantes fumantes e 21 não-fumantes, medindo o IP, IR e $\mathrm{S} / \mathrm{D}$ das artérias uterinas e umbilical, antes e após fumar um cigarro padrão. Não encontraram nenhuma mudança significativa na dopplervelocimetria da artéria uterina e umbilical que pudesse ser atribuída ao efeito agudo do cigarro no grupo de estudo, porém todos os índices da ar- 
téria uterina e a relação S/D da artéria umbilical foram estatisticamente maiores no grupo de fumantes em comparação com o grupo controle, tanto antes quanto após o uso de cigarro. Concluem que o tabagismo crônico causa um aumento na resistência vascular da placenta e do cordão umbilical.

Neste estudo, encontrou-se resultado semelhante, com aumento de resistência vascular tanto na artéria uterina quanto na artéria umbilical, confirmando o efeito crônico do tabagismo. Este aumento de resistência vascular encontrado, no entanto, não alcançou valores patológicos de referência da dopplervelocimetria ${ }^{10,11}$.

A análise da média dos IR das artérias uterinas direita e esquerda (UTM) mostra maior resistência no grupo de fumantes, em todas as semanas analisadas, sendo que na $36^{\text {a }}$ semana de gestação esta diferença foi estatisticamente significante $(p=0,002)$. Possivelmente, se a amostra estudada fosse maior, esta diferença provavelmente seria detectada nas outras semanas de gestação. Isto mostra que o tabagismo na gestação pode ser responsabilizado pelo aumento de resistência na circulação útero-placentária.

Fenômeno semelhante ocorreu quando se analisou a resistência da artéria umbilical, que foi maior no grupo de gestantes fumantes em todas as semanas estudadas, sendo estatisticamente significante na $28^{a}$ semana. O aumento da resistência vascular na circulação feto-placentária das gestantes fumantes, indicando a ação do tabaco na diminuição da oferta de sangue e nutrientes para o feto, está em concordância com o trabalho de Lymperopoulou et al. ${ }^{7}$, que mostraram isoladamente a ação do tabagismo provocando aumento de resistência na artéria umbilical.

Os resultados aqui encontrados mostram o IP da ACM com tendência de ser menor no grupo de fumantes, em concordância com os achados de Abdul-Khaliq et al. ${ }^{12}$. Estes autores, estudando a dopplervelocimetria cerebral em recémnascidos, observaram que nos filhos de mães fumantes há menor resistência na artéria cerebral anterior, artéria carótida interna e artéria basilar.

Tem-se sugerido que quando ocorre hipóxia fetal há centralização de fluxo, refletindo diminuição periférica e aumento na circulação central fetal, efeito denominado de "preservação cerebral"13. Embora o conhecimento destes fatores de ajuste circulatório e seu mecanismo de ação seja incompleto, provavelmente quando a pressão parcial de $\mathrm{O}_{2}$ diminui e a de $\mathrm{CO}_{2}$ se eleva acima de um certo nível, os quimiorreceptores aórticos e carotídeos podem ser ativados, regulando a resposta vasodilatadora central com a finalidade de garantir adequada oxigenação ao cérebro fetal ${ }^{14}$.

$\mathrm{Na}$ análise dos dados encontrados, apesar de os valores dos índices estudados estarem dentro da normalidade, encontrou-se nas gestantes fumantes situação semelhante ao fenômeno de "centralização". Paralelamente à maior resistência na artéria umbilical verificou-se menor resistência na ACM, no grupo de fumantes em relação a não fumantes. Tal fato ficou ainda mais evidente quando da análise da relação IP $\mathrm{ACM} / \mathrm{Umb}$, pois esta mostrou flagrante diferença entre os grupos, sendo a relação sempre menor no grupo de fumantes, estatisticamente significante na $32^{\mathrm{a}}$ e $36^{\mathrm{a}}$ semana de gestação.

Estes dados são preocupantes e servem de alerta para todos os profissionais de saúde, que se interessam pela saúde da gestante e do feto. Ressalta-se a possibilidade de "centralização", fenômeno que ocorre nos fetos com hipóxia crônica, que leva muitas vezes crescimento intrauterino retardado. Mesmo que os valores dos índices estejam dentro da normalidade não invalidam a ressalva e o alerta, pois clinicamente tornam-se importantes estes resultados ao mostrarem, claramente, que o feto tende a ter perfusão sangüínea do tipo de sofrimento crônico.

Evidencia-se, portanto, que o feto de mãe fumante apresenta, graças ao seu nível de carboxi-hemoglobina, uma condição predisponente para hipóxia tecidual, fato que, associado às demais ações do tabaco, podem justificar plenamente os achados de uma tendência à centralização, como o encontrado neste trabalho.

Ressalte-se, ainda, que nesse estudo foram incluídas gestantes sem qualquer patologia. No entanto, devido à alta prevalência do tabagismo, encontrar pacientes com alguma patologia obstétrica e também fumantes é uma situação relativamente freqüente. Nesses casos, o efeito aqui demonstrado da hipóxia crônica para o feto na gestação de mulheres fumantes deve tomar proporções muito maiores e ainda incalculáveis, pois o acréscimo dessa hipóxia oriunda do tabagismo a um sofrimento de base desse feto pode representar o limiar entre a sobrevivência e a morte. Neste estudo detectou-se, pela dopplervelocimetria das artérias uterinas, umbilical e cerebral média, uma alteração com tendência à hipóxia crônica associada a um possivel mecanismo de compensação fetal para manter sua homeostase. 


\section{SUMMARY}

Purpose: to evaluate the influence of cigarette smoking on uteroplacental, fetoplacental and fetal blood flow by obstetric Doppler velocimetry of the uterine, umbilical and middle cerebral arteries.

Method: a prospective study with 42 healthy pregnant women, 20 of whom were smokers and 24 nonsmokers. Ultrasound was performed initially to determine the gestation time. The pregnant women next underwent pulsed and color Doppler velocimetry of the uterine, umbilical and middle cerebral arteries at the 24th, 28th, 32nd, 36th, and 40th week. The smokers were instructed not to smoke for at least two hours before the test.

Results: the mean resistance index (RI) of the right and left uterine arteries was greatest in the smoking group at the 36th week [mean $(S D)=0.501(0.034)],(p=0.002)$. The pulsatility index (PI) of the umbilical artery was greatest in the smoking group at the 28th week [mean (SD) $=1.135(0.182)],(p=0.008)$. No difference in the PI of the middle cerebral artery (MCA) was detected between the two groups. However, the MCA/umbilical PI ratio was lower in the smoking group at the 32nd [mean $(S D)=1.977$ $(0.291)],(p=0.027)$, and 36th week [mean $(S D)=1.850$ $(0.465)],(p=0.014)$.

Conclusion: it was concluded that smoking increases resistance in the uteroplacental and fetoplacental circulation while simultaneously reducing resistance in the MCA, imitating a "brain-sparing" effect, most likely due to chronic hypoxia.

KEY WORDS: Smoking. Normal pregnancy. Dopplervelocimetry.

\section{Referências}

1. Viggiano MG, Caixeta AM, Barbacena ML. Fumo e gravidez: repercussões sobre o concepto e placenta. J Bras Ginecol 1990; 100:147-52.

2. Castles A, Adams EK, Melvin CL, Kelsch C, Boulton ML. Effects of smoking during pregnancy. Five meta-analyses. Am J Prev Med 1999; 16:20815.
3. Westfall TC. Bloqueadores nicotínicos. In: Craig CR, Stitzel RE, editores. Farmacologia Aplicada. São Paulo: Roca; 1986. p.185-93.

4. Longo LD. The biological effects of carbon monoxide on the pregnant woman, fetus, and newborn infant. Am J Obstet Gynecol 1977; 129:69-103.

5. Eriksen PS, Marsal K. Acute effects of maternal smoking on fetal blood flow. Acta Obstet Gynecol Scand 1984; 63:391-7.

6. Morrow RJ, Ritchie JW, Bull SB. Maternal cigarette smoking: the effects on umbilical and uterine blood flow velocity. Am J Obstet Gynecol 1988; 159:1069-71.

7. Lymperopoulou A, Hainaut F, Crimail P, Durand JL, Locatelli C, Maison C. Tabac et grossesse: recherche d'une corrélation cotininémie et Doppler. J Gynecol Obstet Biol Reprod (Paris) 1996; 25:824-7.

8. Bruner JP, Forouzan I. Smoking and buccally administered nicotine. Acute effect on uterine and umbilical artery Doppler flow velocity waveform. J Reprod Med 1991; 36:435-40.

9. Kimya Y, Cengiz C, Ozan H, Kolsal N. Acute effects of maternal smoking on the uterine and umbilical artery blood velocity waveforms. J Matern-Fetal Investig 1998; 8:79-81.

10.Cerri GG, Mólnar LJ, Vezozzo DCP. Aplicação do Doppler em obstetrícia. In: Cerri GG, Mólnar LJ, Vezozzo DCP. Doppler. São Paulo: Sarvier; 1996. p.185-219.

11.Mari G, Deter RL. Middle cerebral artery flow velocity waveforms in normal and small-forgestational-age fetuses. Am J Obstet Gynecol 1992; 166:1262-70.

12.Abdul-Khaliq H, Segerer H, Luck W, Obladem M. Increased cerebral blood flow velocities in newborn infants of smoking mothers. Eur $\mathrm{J}$ Pediatr 1993; 152:232-5.

13.Wladimiroff JW, Tonge HM, Stewart PA. Doppler ultrasound assessment of cerebral blood flow in the human fetus. Br J Obstet Gynaecol 1986; 93:471-5.

14.Dawes GS, Lewis BV, Milligan JE, Roach MR, Talner NS. Vasomotor responses in the hind limbs of foetal and newborn lambs to asphyxia and aortic chemoreceptor stimulation. J Physiol 1968; 195:55-81. 